\title{
Le comportement dipsique
}

Les comportements ont été définis précédemment $\left(\mathrm{m} / \mathrm{s} \quad n^{\circ} 1\right.$, vol. 7 , p. 72) comme le résultat de processus physiologiques qui permettent l'adaptation de l'organisme à son environnement en assurant le maintien de l'homéostasie du milieu intérieur. Le comportement dipsique, ou de prise d'eau, illustre bien cette finalité physiologique dans la mesure où il concourt à maintenir constante la masse liquidienne de l'organisme pour compenser un déficit hydrique.

D'un point de vue comportemental, la soif est une sensation indispensable pour la survie de l'individu, mais les mécanismes qu'elle déclenche le sont rarement par un besoin urgent d'eau. Dans les situations physiologiques, là où l'eau est abondante et les conditions climatiques stables, l'organisme se trouve toujours en bilan hydrique légèrement excédentaire, en particulier chez l'homme où la prise de boisson, par exemple au cours du repas, anticipe toujours sur l'état de déshydratation.

Dans cette fonction contrôlée, le bilan doit prendre en compte les débits d'entrée du compartiment liquidien (prise de boisson, eau apportée par les aliments et eau métabolique) et les débits de sortie (élimination urinaire et sudation, d'un point de vue physiologique ; éventuellement diarrhée et hémorragie, d'un point de vue pathologique). Les multiples éléments qui interviennent dans la chaîne de régulation tiennent compte du fait que l'eau corporelle se répartit en deux compartiments : extracellulaire (CEC, composé du liquide interstitiel et du liquide vasculaire circulant) et intracellulaire (CIC). C'est pourquoi, d'un point de vue physiologique, on parle de "soif $\mathrm{m} / \mathrm{s} n^{\circ} 2$, vol. 7 , février 91 extracellulaire " et de "soif intracellulaire".

Le comportement dipsique est l'élément régulateur du débit d'entrée d'eau dans les compartiments liquidiens, plus précisément dans le compartiment extracellulaire. Un autre facteur va régler les échanges d'eau entre les compartiments intra- et extracellulaires, la pression osmotique qui résulte de la différence de concentration des ions entre ces deux compartiments (principalement $\mathrm{Na}+$ à l'extérieur et $\mathrm{K}+$ à l'intérieur de la cellule), différence qui doit rester constante tout au long de la vie de l'organisme. L'eau diffuse entre les deux compartiments en fonction de la pression osmotique, le flux étant orienté du compartiment le plus dilué vers le compartiment le plus concentré. L'apport alimentaire de sels intervient donc directement dans la régulation du comportement dipsique. Il se caractérise par un comportement spécifique, couplé au comportement dipsique, l'appétit pour le sel.

La déshydratation intracellulaire, ou " soif intracellulaire ", est détectée par des neurones, récepteurs sensibles à la pression osmotique, situés dans l'hypothalamus antérieur, l'" aire de la soif "découverte par Andersson en 1952. Dans une première série d'expériences, il a stimulé une toute petite zone de l'hypothalamus d'une chèvre vigile libre de ses mouvements avec des solutions de $\mathrm{NaCl}$, et déclenché un comportement dipsique. Il a précisé la région concernée en effectuant une microstimulation électrique de la même zone hypothalamique et il a montré qu'une telle stimulation déclenchait une séquence comportementale intégrée, "l'animal s'engageant dans un comportement dipsique qui se prolongeait tant que la stimulation était maintenue ". Il a ainsi délimité cette aire de la soif entre la colonne antérieure du fornix et le faisceau mamillo-thalamique ou faisceau de Vicq d'Azyr (figure 1, p. 178). L'aire de la soif peut ainsi être considérée comme étant à l'origine d'un réflexe dipsique non conditionné.

La déshydratation extracellulaire, ou " soif extracellulaire ", est détectée par plusieurs types de récepteurs mettant en jeu deux boucles régulatrices, l'une associée à une voie de communication purement nerveuse et l'autre faisant intervenir des intermédiaires endocriniens. Cette multiplicité de systèmes de réception engendre plusieurs types de messages nerveux et hormonaux qui sont intégrés au niveau central pour commander le déclenchement du comportement permettant de compenser le déficit détecté.

La première boucle est mise en jeu à partir de récepteurs volémiques, c'est-à-dire sensibles à la variation du volume sanguin. Les uns sont situés au niveau de l'atrium cardiaque et les autres au niveau de l'hypothalamus antérieur, près de la zone des récepteurs sensibles à la pression osmotique. En cas de diminution de la volémie, un message nerveux codant pour cette information est transmis vers l'aire hypothalamique de la soif, via le système nerveux périphérique et le bulbe dans le cas des récepteurs atriaux, et le comportement dipsique est déclenché. Dans le cas contraire, une augmentation de la volémie engendrera un message codant pour la diminution voir l'arrêt de la prise de boisson.
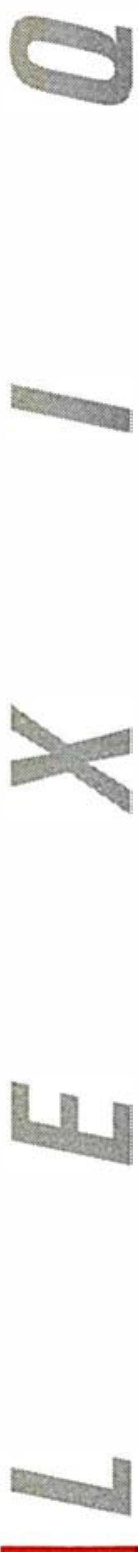


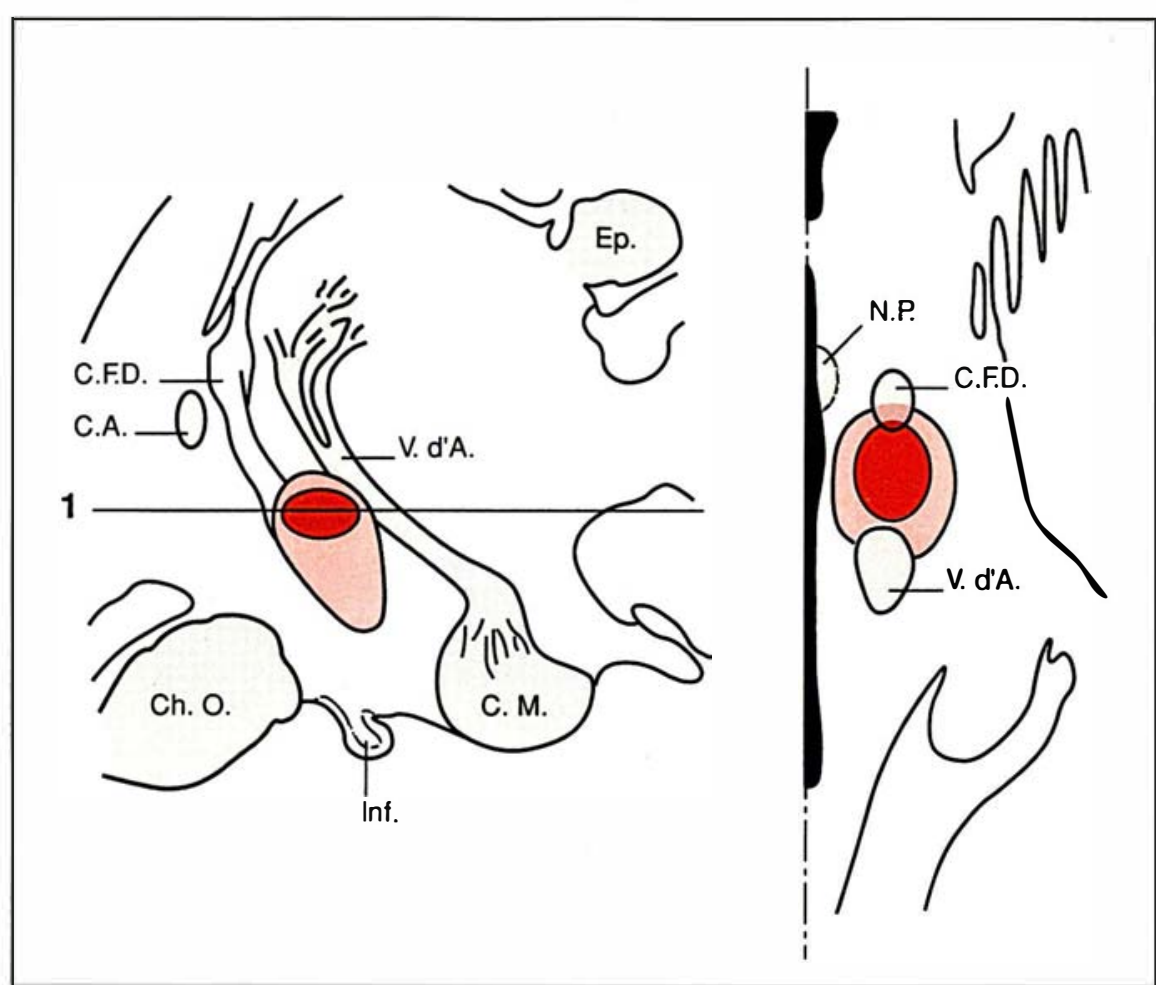

Figure 1. L'aire hypothalamique de la soif selon Andersson. A gauche: section sagittale de l'encéphale de chèvre montrant les régions où des microinjections de $\mathrm{NaCl}$ déclenchent le comportement dipsique (rose) ainsi que les régions, plus restreintes, où des stimulations électriques déclenchent ce même comportement (rouge). A droite : demi-coupe horizontale (plan 1 de la coupe sagittale) montrant les mêmes régions. C.M. : corps mammillaires; Ch.O. : chiasma optique ; Inf. : infundibulum ; V. d'A. : faisceau de Vicq d'Azyr ; C.A. : commissure antérieure; Ep. : epiphyse; N.P. : noyau paraventriculaire ; C.F.D. : columna fornicis descendens. (D'après Andersson et McCann, Acta Physiol Scan $1957 ; 41$ : 194-8.)

La deuxième boucle est mise en jeu à partir de deux types de récepteurs périphériques. Les premiers sont situés dans l'atrium cardiaque dont la distension par accroissement de la volémie libère de l'hormone atriale natriurétique (atrial natriuretic factor, ANF). Cette hormone a une double action : rénale, favorisant la diurèse et la natriurèse, et nerveuse, inhibant par un mécanisme central, le comportement dipsique et l'appétit pour le sel. Les seconds sont situés au niveau de l'appareil juxtaglomérulaire rénal. Les cellules granulaires des parois des artérioles afférentes à cet appareil synthétisent et sécrètent une protéine enzymatique, la rénine. Ces cellules sont elles-mêmes des récep- cepteurs intrarénaux) et sont stimulées pour la sécrétion de rénine par une diminution de la pression artérielle. Dans la zone tubulaire distale juxtaglomérulaire, appellée macula densa, se trouvent des récepteurs sensibles à la concentration en $\mathrm{Na}+$ et en $\mathrm{Cl}^{-}$dans les fluides tubulaires : une diminution de ces concentrations stimule la sécrétion de rénine. Celleci initie la synthèse de l'angiotensine II (AT II) à partir de l'angiotensinogène, protéine plasmatique circulante. L'AT II, outre son rôle périphérique hypertenseur, peut, comme l'ANF, passer la barrière hématoencéphalique dans des zones très limitées de l'encéphale, par exemple au voisinage de l'organe vasculaire de la lame terminale. Au niveau de cette structure, elle exerce un puissant effet dipsogène lorsqu'elle est injectée en très petites quantités au moyen d'une canule intracérébrale, l'ANF ayant un effet opposé. L'AT II peut également être synthétisée par le tissu nerveux central. Des neurones situés dans la paroi du troisième ventricule, au voisinage des volorécepteurs hypothalamiques (cf. ci-dessus), ont leur activité stimulée par l'AT II ; injectée en très petites quantités à ce niveau, elle déclenche le comportement dipsique.

Enfin il existe une régulation sensorielle de l'étanchement à partir de la langue et de l'estomac: ces tissus possèdent des récepteurs engendrant des messages nerveux qui, transmis à l'aire antérieure de l'hypothalamus, provoquent un étanchement provisoire pendant le temps nécessaire à l'eau ingérée pour pénétrer dans le compartiment extracellulaire.

L'ensemble des facteurs physiologiques qui contrôlent le comportement dipsique est donc fortement couplé à la régulation homéostasique hydrominérale. Mais d'autres facteurs interviennent dans la régulation de ce comportement, comme l'association avec le caractère agréable d'une boisson (par exemple, l'addition de saccharose dans l'eau augmente la quantité de boisson absorbée chez le rat) ou, au contraire, avec son caractère désagréable. Chez l'homme, ce caractère est renforcé par un facteur social qui fait jouer à la boisson un rôle de vecteur relationnel gratifiant renforçant la prise de boisson en dehors de tout déséquilibre physiologique générateur d'une sensation de soif.

Un comportement aussi "élémentaire " que le comportement dipsique résulte donc de l'intervention contrôlée d'un ensemble intégré de facteurs neuro-endocriniens ; si une structure nerveuse centrale peut jouer un rôle prépondérant (ici l'aire hypothalamique de la soif), elle n'en est pas pour autant "le centre " qui ferait d'elle l'unique responsable d'un comportement donné. 